\title{
Persistent contaminants in waste oils: a short review on PCBs and PAHs as main contaminants
}

\begin{abstract}
Persistent organic pollutants (POPs) include organochlorine compounds such as polychlorinated biphenyls (PCBs). Polycyclic aromatic hydrocarbons (PAHs) are considered as POP-like chemicals because of their similar characteristic. One source of entry of PCBs into the environment is from the improper handling and disposal of PCB-contaminated waste oil from old or discarded transformers. Polychlorinated hydrocarbons are natural constituents of transformer oils and are essential in prolonging transformer in-service lifetime. The existence of PCBs and PAHs in waste oils is reported in different studies. The aim of this review paper is to investigate the source and level of such pollutants like PCBs and PAHs in all kinds of oils with more attention to waste motor oil.
\end{abstract}

Keyword: Waste oil; Transformer oil; PCBs; PAH 